\title{
Successful Conversion Surgery for Stage IV Gastric Cancer after Nivolumab Monotherapy as Third-Line Chemotherapy
}

\author{
Hayato Watanabe ${ }^{a}$ Hirohito Fujikawa ${ }^{a}$ Keisuke Komori ${ }^{a}$ Kazuki Kano ${ }^{a}$ \\ Kosuke Takahashia Takanobu Yamada ${ }^{a}$ Yasuhiro Inokuchi ${ }^{b}$ \\ Nozomu Machidab Tomoyuki Yokose ${ }^{c}$ Yasushi Rino $^{d}$ \\ Munetaka Masuda ${ }^{d}$ Takashi Ogata $^{a}$ Takashi Oshima ${ }^{a}$ \\ aDepartment of Gastrointestinal Surgery, Kanagawa Cancer Center, Yokohama, Japan; \\ bDepartment of Gastroenterology, Kanagawa Cancer Center, Yokohama, Japan; \\ 'Department of Pathological Diagnosis, Kanagawa Cancer Center, Yokohama, Japan; \\ dDepartment of Surgery, Yokohama City University, Yokohama, Japan
}

\author{
Keywords \\ Conversion surgery · Gastric cancer · Nivolumab
}

\begin{abstract}
There are few reports of conversion surgery (CS) after nivolumab monotherapy because it is considered as a third-line standard chemotherapy for unresectable or recurrent gastric cancer. Here, we report a rare case of stage IV gastric cancer effectively treated with CS after nivolumab monotherapy as a third-line chemotherapy. A 73-year-old man was referred to our hospital with loss of appetite and abdominal discomfort. Stage IV gastric cancer with liver metastasis was diagnosed via upper gastrointestinal endoscopy and CT. Twelve courses of capecitabine, cisplatin, and trastuzumab were administered as the first-line treatment, 25 courses of paclitaxel plus ramucirumab as the second-line treatment, and 31 courses of nivolumab monotherapy as the third-line treatment. After 31 courses of nivolumab monotherapy, CT showed that the primary tumor shrank with no liver metastasis or ascites. Diagnostic laparoscopy was performed with no peritoneal dissemination (P0), and the peritoneal lavage cytology was negative (CY0). CS was performed with total gastrectomy and D2 lymph node dissection (R0 resection). The pathological diagnosis was $U$, Ant-Less, Type 2, $70 \times 63$ $\mathrm{mm}$, poorly differentiated adenocarcinoma (ypT3NOM0 ypStage IIA). R0 resection was performed, and the histological response was grade 1a. The patient did not show recurrence for 9 months after CS.
\end{abstract}


Watanabe et al.: Successful Conversion Surgery for Gastric Cancer after Nivolumab Monotherapy

\section{Introduction}

Nivolumab monotherapy is considered the third-line standard chemotherapy for unresectable or recurrent gastric cancer [1,2]. There are few reports on conversion surgery (CS) after nivolumab monotherapy. CS is often performed when first-line chemotherapy is effective, and although the response rate of nivolumab monotherapy is not high, it is effective after the third-line chemotherapy in some cases. Thus, long-term survival and the number of patients undergoing CS after nivolumab as third-line chemotherapy are expected to increase in the future. Here, we report a rare case of stage IV gastric cancer effectively treated with CS after nivolumab monotherapy as the third-line chemotherapy.

\section{Case Presentation}

A 73-year-old man was referred to our hospital because of loss of appetite and abdominal discomfort. Upper gastrointestinal endoscopy showed a type 3 advanced gastric cancer, occupying the anterior wall and lesser curvature side of the upper part of the stomach (Fig. 1a). The tumor was diagnosed as poorly differentiated adenocarcinoma by pathological examination of biopsy, and immunohistochemistry for human epidermal growth factor receptor 2 (HER2) showed a score of 3+ (Fig. 1b). CT revealed an approximately 80-mm diameter tumor with obvious blurring at the anterior wall, indicating serosal invasion, enlarged lymph nodes around the lesser curvature, and a 27-mm diameter metastatic liver tumor in segment 8 of the liver (Fig. 1c, d). Thus, the clinical diagnosis was U type 3, cT4aN2M1 (HEP) cStage IV according to the eighth edition of the Union for International Cancer Control TNM Classification [3].

The treatment plan was chemotherapy. Considering that the tumor was HER2 positive, capecitabine $(2,400 \mathrm{mg} /$ day $)$, cisplatin $\left(40 \mathrm{mg} / \mathrm{m}^{2} /\right.$ day $)$, and trastuzumab $\left(8 \mathrm{mg} / \mathrm{m}^{2} /\right.$ day) were administered as the first-line chemotherapy. After 12 courses, CT showed an increased primary tumor with shrunken liver metastasis. According to the Response Evaluation Criteria in Solid Tumors (RECIST) version 1.1 guidelines, the tumor was classified as progressive disease. Paclitaxel $\left(80 \mathrm{mg} / \mathrm{m}^{2} /\right.$ day on day 1$)$ combined with ramucirumab ( $8 \mathrm{mg} / \mathrm{kg} /$ day on day 1 ) was administered as the second-line chemotherapy. After 25 courses, CT showed ascites with no progression of the primary and metastatic liver tumors. The objective tumor response was classified as progressive disease. Nivolumab monotherapy ( $240 \mathrm{mg} /$ body infused on day 1 and every 2 weeks) was administered as the third-line chemotherapy. After 31 courses of nivolumab monotherapy, CT showed a significantly shrunken primary tumor, with no liver metastasis or ascites (Fig. 2a, b). There was no significant distant metastasis on PET-CT. According to the RECIST guidelines, the tumor response was classified as partial response. Diagnostic laparoscopy was performed to exclude peritoneal dissemination. There was no peritoneal dissemination (P0), and peritoneal lavage cytology was negative (CY0). Considering the aforementioned findings, the diagnosis after nivolumab monotherapy was ycT3N0M0 ycStageIIA (TNM8th), and CS (curative gastrectomy with D2 lymph node dissection) was determined.

The patient underwent CS (curative gastrectomy with D2 lymph node dissection) and was discharged on postoperative day 8 with no complications. The pathological diagnosis was U, Ant-Less, Type 2, $70 \times 63 \mathrm{~mm}$, poorly differentiated adenocarcinoma, ypT3N0M0 ypStageIIA (Fig. 3). The pathological response was grade 1a. After 43 months of first-line chemotherapy, the patient is alive with no signs of recurrence 9 months postoperatively.

\section{Karger'}


Watanabe et al.: Successful Conversion Surgery for Gastric Cancer after Nivolumab Monotherapy
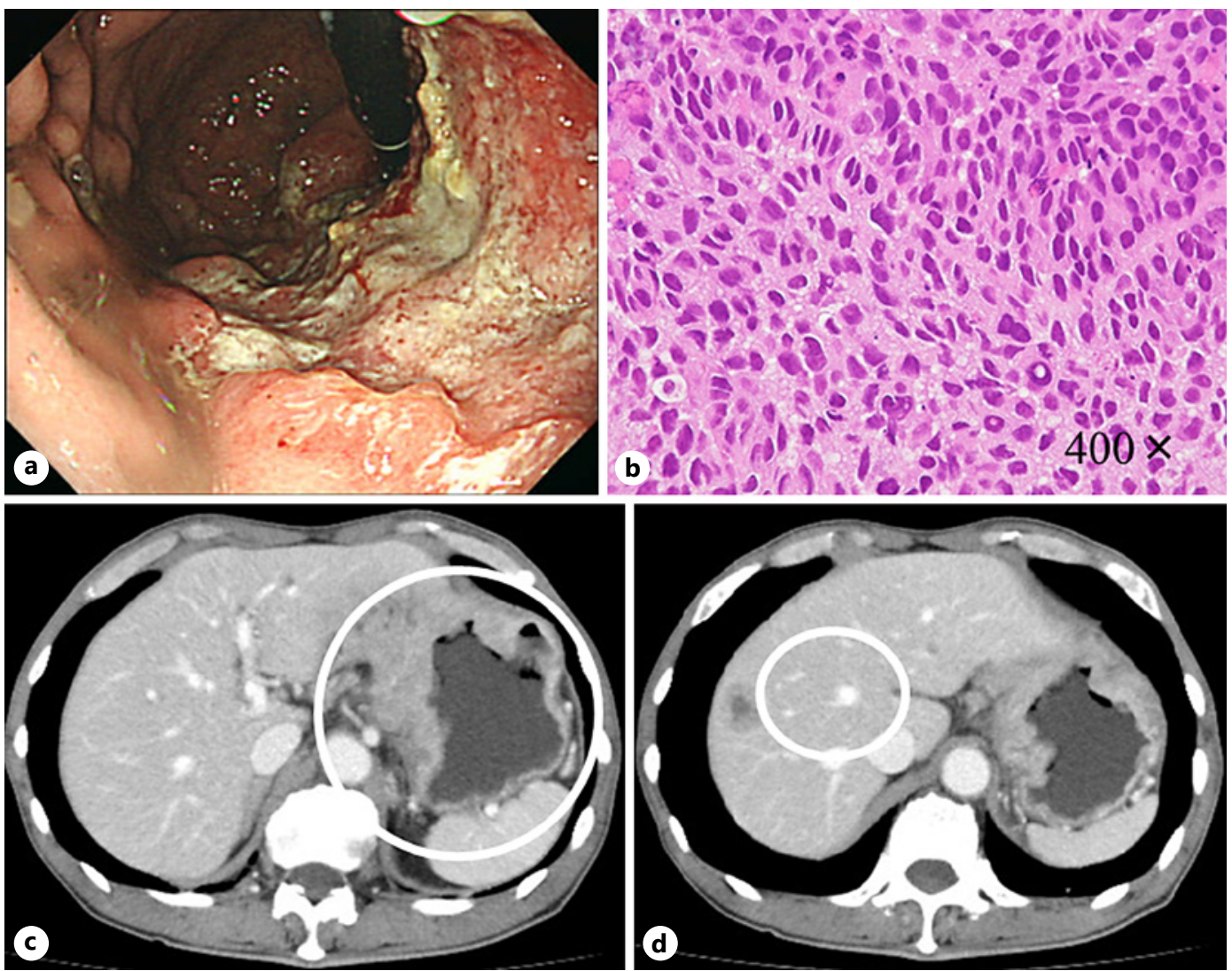

Fig. 1. a Upper gastrointestinal endoscopy revealing type 3 advanced gastric cancer, occupying the anterior wall and lesser curvature of the upper part of the stomach. $\mathbf{b}$ Biopsy confirms the diagnosis of adenocarcinoma (poor) with a glandular duct structure (magnification, $\times 400$ ). c CT shows an approximately $80-\mathrm{mm}$ diameter transmural tumor with obvious blurring at the anterior wall and lesser curvature of the upper part of the stomach and enlarged lymph nodes surrounding the lesser curvature (circle). d A 27-mm metastatic liver tumor at segment 8 of the liver is detected on CT (circle).
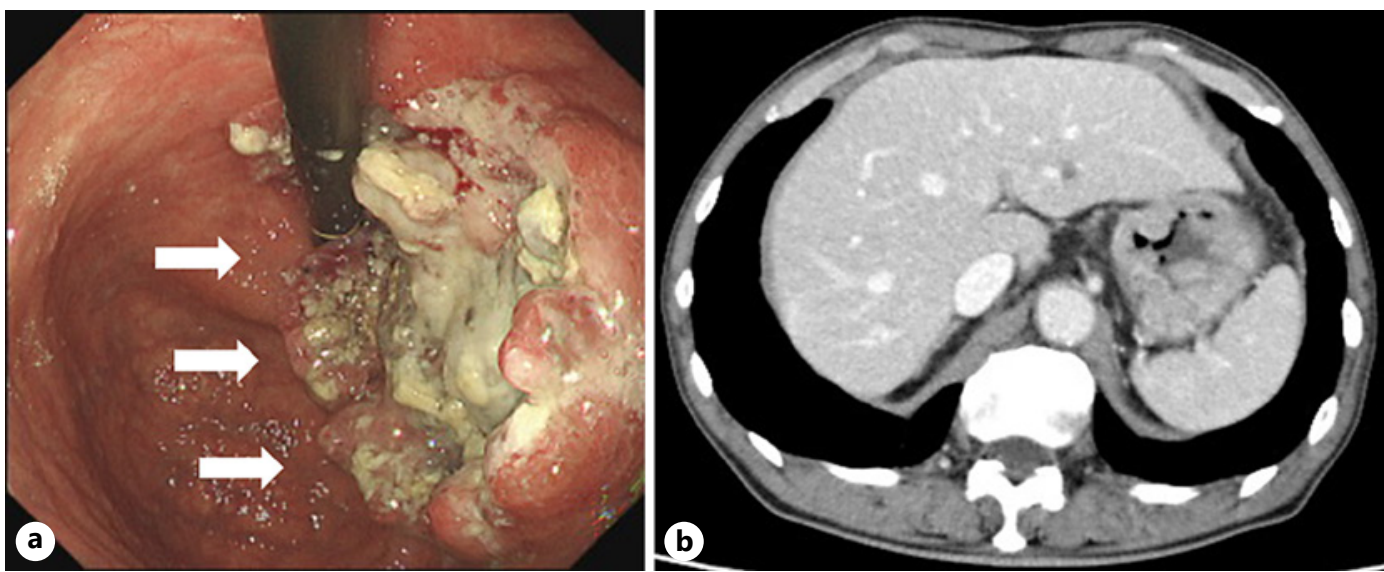

Fig. 2. a Upper gastrointestinal endoscopy after third-line chemotherapy showing that the primary tumor has shrunken (arrows). b CT showing the shrunken primary tumor and liver metastasis. 
Watanabe et al.: Successful Conversion Surgery for Gastric Cancer after Nivolumab Monotherapy

Fig. 3. The pathological diagnosis is $U$, AntLess, Type 2, $70 \times 63 \mathrm{~mm}$, poorly differentiated adenocarcinoma, ypT3N0M0 ypStageIIA.

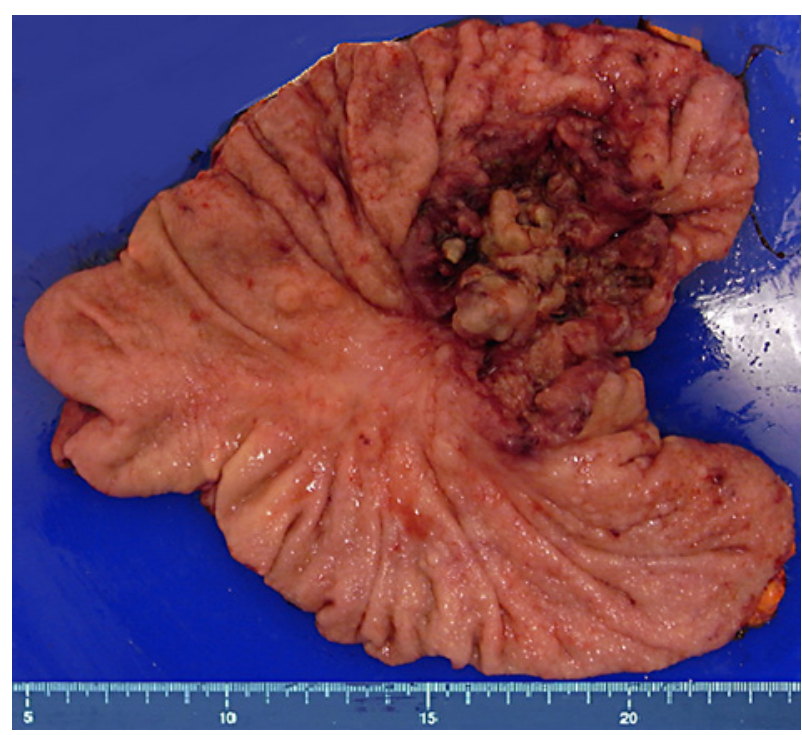

\section{Discussion/Conclusion}

Nivolumab enhances antitumor T-lymphocyte activity by blocking immune checkpoint inhibitors of programmed death-1 [4]. Programmed death-1 is a protein expressed on the surface of lymphocytes and negatively regulates their immune activity [5, 6]. In 2017, the ATTRACTION-2 study demonstrated the efficacy of nivolumab monotherapy in patients with advanced gastric cancer or gastroesophageal junction cancer [5]. In that study, the 2-year overall survival rates were 10.6 and $3.2 \%$ in the nivolumab and placebo groups, respectively. Furthermore, the 2-year follow-up indicated that objective response rates were 11.9 and $0 \%$ in the nivolumab and placebo groups, respectively [7], and the overall response rate of nivolumab was only $11.2 \%$, which was not high. To the best of our knowledge, there are only 3 reports on cases of stage IV gastric cancer successfully treated with curative resection after third-line chemotherapy with nivolumab monotherapy [8-10].

In this patient, liver metastasis was the stage IV factor. The rate of liver metastasis is reported to be $4-14 \%$ in stage IV gastric cancer [11-14]. For stage IV gastric cancer with liver metastases, systemic chemotherapy is recommended because surgical removal of liver metastases of gastric cancer is still controversial, and in most cases, multiple recurrences of the liver metastases can occur after hepatectomy $[15,16]$. Although CS is an option for unresectable, advanced, or metastatic gastric cancer when distant metastases are controlled [16], little or no prospective trial investigating systemic chemotherapy specified in liver metastases has been reported. Moreover, the usefulness of CS for liver metastases with gastric cancer remains unsatisfactory.

The optimal timing for CS remains controversial, and its indications need to be clarified. Several reports stated that decreased serum tumor marker levels could be associated with clinical response $[17,18]$. In this patient, the cancer antigen 125 level increased upon the initiation of nivolumab monotherapy but decreased dramatically thereafter. After 24 courses, serum tumor marker levels decreased to normal, and CT revealed that the primary tumor shrank, with no liver metastasis or ascites. The treatment was continued for 3 months (additional 7 courses), and CS was decided after confirming that there was no recurrence via CT and PET/CT. Further verification is required regarding the optimal timing and indications for CS. The 2-year follow-up results of the previous ATTRACTION-2 study showed that the 2-year survival rate of 32 patients, including complete remission (CR) and partial response, was 
good at $61.3 \%$. Therefore, it is controversial whether nivolumab should be continued or whether CS should be performed. In this case, CR was achieved for the metastatic tumor, and the primary tumor was shrunken; however, as CR was not achieved for the primary tumor, CS was performed.

We report a rare case of stage IV gastric cancer successfully treated with CS after thirdline chemotherapy with nivolumab monotherapy. Further reports of similar cases are expected in the future.

\section{Statement of Ethics}

This case report was exempted from the Institutional Review Board of the Kanagawa Cancer Center as per its policy. Written informed consent was obtained from the patient for publication of this case report and any accompanying images.

\section{Conflict of Interest Statement}

None of the authors have any financial conflicts of interest to declare. All authors have confirmed that the article is not under consideration for review by any other journal.

\section{Funding Sources}

No funding was available for this study.

\section{Author Contributions}

The initial version of the manuscript was prepared by Watanabe et al. The patient's diagnosis and care were performed by Komori Keisuke, Kano Kazuki, Takahashi Kosuke, Yamada Takanobu, Inokuchi Yasuhiro, Machida Nozomu, Suzuki Masaki, Yokose Tomoyuki, Ogata Takashi, and Oshima Takashi. Drafting the article and preparation of figures were carried out by Watanabe Hayato, Fujikawa Hirohito, and Oshima Takashi. The article and the edited final version of the manuscript were revised by Watanabe Hayato, Fujikawa Hirohito, and Oshima Takashi. Finally, this article was reviewed before submission by Rino Yasuhi and Masuda Munetaka and all other 12 investigators. Thus, all authors actively participated in this study.

\section{References}

1 Kang YK, Boku N, Satoh T, Ryu MH, Chao Y, Kato K, et al. Nivolumab in patients with advanced gastric or gastrooesophageal junction cancer refractory to, or intolerant of, at least two previous chemotherapy regimens (ONO-4538-12, ATT RAC TION-2): a randomised, double-blind, placebo-controlled, phase 3 trial. Lancet. 2017 Dec;390(10111):2461-71.

2 Japanese Gastric Cancer Association. Japanese classification of gastric carcinoma. 15th ed. Tokyo: Kanehara; 2017; in Japanese.

3 Brierley J, Gospodarowicz M, Wittekind C. TNM classification of malignant tumours. 8th ed. New York: WileyBlackwell; 2017.

4 Sharma P, Allison JP. Immune checkpoint targeting in cancer therapy: toward combination strategies with curative potential. Cell. 2015 Apr;161(2):205-14.

5 Nishimura H, Minato N, Honjo T. Immunological studies on PD-1 deficient mice: implication of PD-1 as a negative regulator for B cell responses. Int Immunol. 1998 Oct;10(10):1563-72. 
6 Topalian SL, Drake CG, Pardoll DM. Immune checkpoint blockade: a common denominator approach to cancer therapy. Cancer Cell. 2015 Apr;27(4):450-61.

7 Chen LT, Satoh T, Ryu MH, Chao Y, Kato K, Chung HC, et al. A phase 3 study of nivolumab in previously treated advanced gastric or gastroesophageal junction cancer (ATTRACTION-2): 2-year update data. Gastric Cancer. 2020;23(3):510-9.

8 Shiraishi T, Kano M, Sakata H, Murakami K, Toyozumi T, Sekino N, et al. [Successful treatment of gastric cancer with conversion surgery after nivolumab treatment: a case report]. Gan To Kagaku Ryoho. 2019 Oct;46(10): 1614-6.

9 Toyota S, Orita H, Fukuyama Y, Motoyoshi S, Kawanami S, Maeda S, et al. Successful conversion surgery following chylous ascites after nivolumab for advanced gastric cancer. In Vivo. 2020 Mar-Apr;34(2):583-5.

10 Matsumoto R, Arigami T, Matsushita D, Okubo K, Tanaka T, Yanagita S, et al. Conversion surgery for stage IV gastric cancer with a complete pathological response to nivolumab: a case report. World J Surg Oncol. 2020 Jul;18(1):179.

11 Koga S, Kawaguchi H, Kishimoto H, Tanaka K, Miyano Y, Kimura O, et al. Therapeutic significance of noncurative gastrectomy for gastric cancer with liver metastasis. Am J Surg. 1980 Sep;140(3):356-9.

12 Okuyama K, Isono K, Juan IK, Onoda S, Ochiai T, Yamamoto Y, et al. Evaluation of treatment for gastric cancer with liver metastasis. Cancer. 1985 May;55(10):2498-505.

13 Sakamoto Y, Ohyama S, Yamamoto J, Yamada K, Seki M, Ohta K, et al. Surgical resection of liver metastases of gastric cancer: an analysis of a 17-year experience with 22 patients. Surgery. 2003 May;133(5):507-11.

14 Takemura N, Saiura A, Koga R, Arita J, Yoshioka R, Ono Y, et al. Long-term outcomes after surgical resection for gastric cancer liver metastasis: an analysis of 64 macroscopically complete resections. Langenbecks Arch Surg. 2012 Aug;397(6):951-7.

15 Kinoshita T, Saiura A, Esaki M, Sakamoto H, Yamanaka T. Multicentre analysis of long-term outcome after surgical resection for gastric cancer liver metastases. Br J Surg. 2015 Jan;102(1):102-7.

16 Oki E, Tokunaga S, Emi Y, Kusumoto T, Yamamoto M, Fukuzawa K, et al. Surgical treatment of liver metastasis of gastric cancer: a retrospective multicenter cohort study (KSCC 1302). Gastric Cancer. 2016 Jul;19(3):96876.

17 Yamaguchi K, Yoshida K, Tanahashi T, Takahashi T, Matsuhashi N, Tanaka Y, et al. The long-term survival of stage IV gastric cancer patients with conversion therapy. Gastric Cancer. 2018 Mar;21(2):315-23.

18 Passler M, Taube ET, Sehouli J, Pietzner M. Pseudo-or real progression? An ovarian cancer patient under nivolumab: a case report. World J Clin Oncol. 2019 Jul;10(7):247-55. 